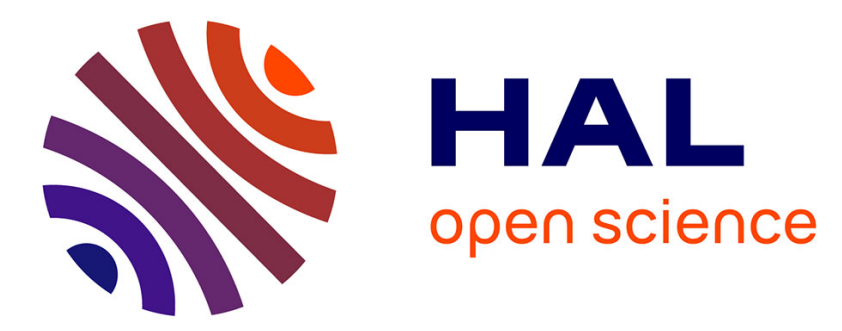

\title{
OPTOGALVANIC DETECTION WITH MICROWAVE DISCHARGE
}

\author{
T. Suzuki, H. Sekiguchi, T. Kasuya
}

\section{To cite this version:}

T. Suzuki, H. Sekiguchi, T. Kasuya. OPTOGAlvANIC DETECTION WITH MICROWAVE DISCHARGE. Journal de Physique Colloques, 1983, 44 (C7), pp.C7-419-C7-427. 10.1051/jphyscol:1983740 . jpa-00223297

\section{HAL Id: jpa-00223297 https://hal.science/jpa-00223297}

Submitted on 1 Jan 1983

HAL is a multi-disciplinary open access archive for the deposit and dissemination of scientific research documents, whether they are published or not. The documents may come from teaching and research institutions in France or abroad, or from public or private research centers.
L'archive ouverte pluridisciplinaire HAL, est destinée au dépôt et à la diffusion de documents scientifiques de niveau recherche, publiés ou non, émanant des établissements d'enseignement et de recherche français ou étrangers, des laboratoires publics ou privés. 


\title{
OPTOGALVANIC DETECTION WITH MICROWAVE DISCHARGE
}

\author{
T. Suzuki, H. Sekiguchi ${ }^{*}$ and T. Kasuya \\ The Institute of Physical and Chemical Research, Wako, Saitama 351, Japan \\ *Toyo University, Kawagoe, Saitama 356, Japan
}

\begin{abstract}
Résumé - La détection optogalvanique dans une décharge micro-onde est démontrée avec un rapport $\mathrm{S} / \mathrm{N}$ raisonnablement haut en utilisant un magnétron. La méthode utilisée à double sonde se caractérise par les deux points suivants : - un haut rendement d'extraction des signaux $O G$, et un fonctionnement stable dans un large domaine de pression (1-40 Torr pour l'hêlium). Ces caractéristiques sont bien mises en évidence par l'observation de transitions moléculaires de 1'hélium. Un mécanisme simple de formation des signaux dans la méthode à double sonde est aussi présenté.
\end{abstract}

Abstract - The optogalvanic detection in a microwave discharge is demonstrated with a reasonably high $S / N$ ratio, using a magnetron. The employed double-probe method is characterized by two aspects; a high extraction efficiency of the optogalvanic signal and a stable operation in a wide pressure range (1-40 Torr for Helium). These features are demonstrated well by the observation of molecular transitions of Helium. A mechanism of the signal generation in the double-probe method is also discussed.

\section{Introduction}

Optogalvanic spectroscopy has proved to be a powerful technique for the study of molecular species in a flame [1] or in a discharge plasma [2-7]. While most of the studies of optogalvanic effect (OGE) have used a hollow-cathode dc discharge, the detection method with electrodeless high frequency discharges has been demonstrated to be usefu] especially for high resolution study $[5,8]$ and for studying unstable molecules [6].

Ionization and electron-loss mechanisms in gas discharges are varied with the frequency of the applied field [9]. Ion and electron motions are different for high-requency discharges sustained by $r f$ and microwave fields. In a typical glow discharge, electrons experience many collisions in a period of oscillation in radio frequency $(r f)$ field. In contrast, electrons experience many oscillaitons in a period of collision in microwave field. In respect of the OG mechanism, in which the collisional ionization is one of main processes, the $0 G$ signals obtained in these different types of discharge are expected to provide valuable informations. Furthermore, high-frequency discharges are used in many fields for a creation of free radicais. For the study of chemical processes involved in these discharges, the optogalvanic method is expected to be a useful technique of detection.

Several kinds of experimental technique developed for the NMR and ESR spectroscopy are readily available for the optogalvanic detection with the microwave discharge. In Ar discharge sustained by a magnetron oscillator the OG signals can be observed through the laser-induced variation of the reflected microwave power or through the changes of the anode current of the magnetron. But, these methods were not practical because of inherent large noises of magnetron. Instead, we adopted the following method in which the $O G$ signals were extracted through two probes placed in contact with the discharge plasma just like the double-probe method used 
in the plasma diagnostics. The double-probe method with rf discharge was recently applied for the study of the Rydberg states of xenon by Labastie et a1. [10].

In this paper, mechanistic study of the $O G$ signals extracted by the double probe in the microwave discharge is presented together with a spectroscopic application to $\mathrm{He}_{2}$ molecule.

\section{Experiments with double probes}

Figure 1 shows a schematic of the experimental arrangement used to record the OG spectra. The experimental set-up is the same as described in [3] except the signal collecting networks described below. A commercial magnetron is operated with a current regulating circuit. The microwave output was fed into the microwave cavity (Evenson type) through a coaxial cable. The reflected microwave was extracted by a directional coupler and its power was monitored by a microwave diode ( 1N2iC). The microwave power was variable only within a limited range around $20 \mathrm{~W}$ where the magnetron had the least noises. We used a pyrex tube of $13 \mathrm{~mm} 0 \mathrm{D}$. The gas is continuously pumped through the discharge, and the pressure is measured by a capacitance manometer (MKS, Baratron). A CW dye laser (Coherent, CR-599-21) is operated mostly on multimodes with maximum output of $300 \mathrm{~mW}$. The laser beam is mechanically chopped at $220 \mathrm{~Hz}$ and focused into the discharge. The laser wavelength is scanned with a gear-coupled stepping motor which rotates the micrometer drive of a birefringent filter. Two probes made of tungsten wire of $0.5 \mathrm{~mm}$ diameter are placed inside the cel1. Figure 2 shows the double probes of two configurations; parallel rings and parallel rods. When the discharge is sustained, potential difference of several volts appears across the two probes due to the inhomogeneous electric field inside the cavity. The laser-induced voltage chages are measured using an appropriate load resistance of $100 \mathrm{k} \Omega$ or $1 \mathrm{M} \Omega$. A time constant of the lock-in amplifier was $0.3 \mathrm{~s}$.

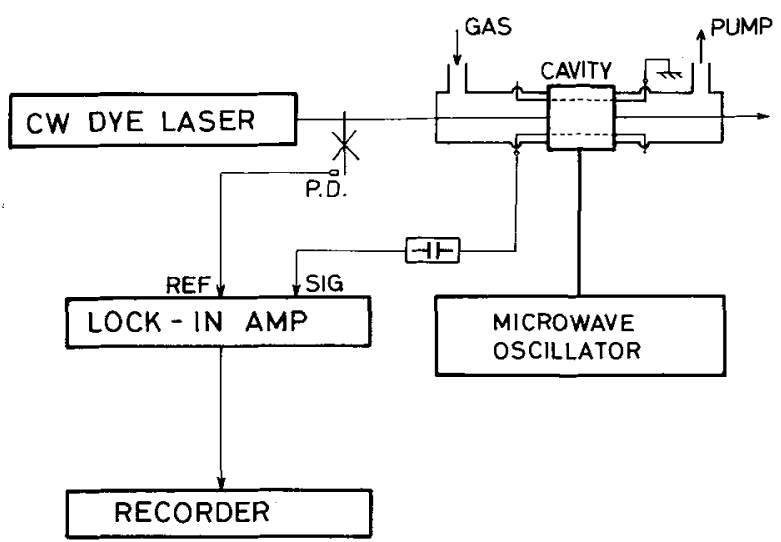

Fig. 1 Schematic drawing of the apparatus used in the optogalvanic double-probe detection.

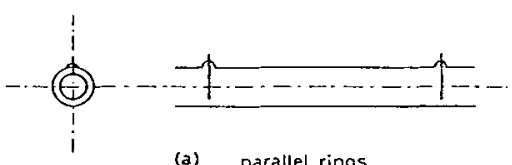

(a) parallel rings

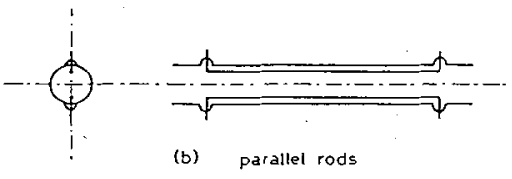

Fig. 2 Two types of double probes.

Typical examples of the Ar spectra are shown in Fig. 3, which were recorded with three different methods; (a) by measuring the reflected microwave power, and using double probes of (b) parallel rings and (c) parallel rods. As the magnetron has very large noises compared with other oscillators such as a klystron or a Gunn diode, the method of measuring the reflected microwave power is not practical for the OGE detection as shown in Fig. 3(a). The $S / N$ ratio obtained with the double probe of parallel rods is two orders of magnitude larger than that obtained with parallel rings. There are some differences in operation between the two configura- 


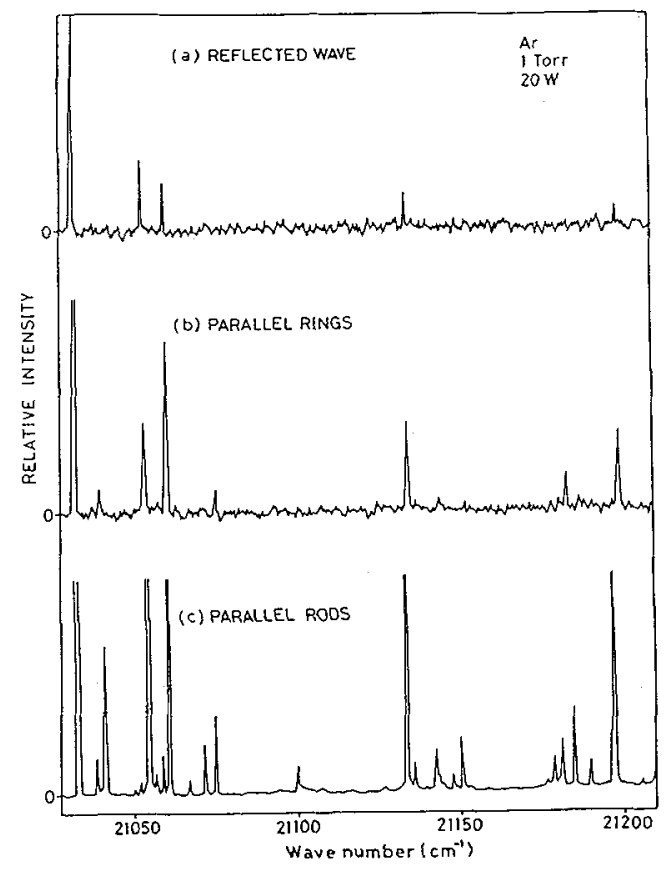

Fig. 3 The optogalvanic spectra of argon recorded by (a) measuring the reflected microwave power, and using double probes of (b) parailel rings and (c) parallel rods.

tions of the double probe. First, the probes are in close contact with a discharge plasma in paraliel rods. Secondly, the laser beam can be passed along the parallel. rods. Finally, in the parallel-ring configuration, the positive and negative $O G$ signals are partially cancelled as will be discussed in a later section. These features favor the parallel-rod configuration as the double probe in the OGE detection. Therefore, only the double probe of parallel rods are used hereafter.

Although noises in Fig. 3(c) appear to be small, they are still caused by the magnetron. This is confirmed by the following experiment. With an external modulation of the microwave amplitude, we obtain a modulated voltage induced across the two probes. The extrapolation of the modulation depth to the noise level of the magnetron oscillator gives an approximate noise voltage shown in Fig. 3(c). Therefore the large signal to noise ratio obtained with the double probe of parallel rods is resulted from a high efficiency in extracting the OG signals (high sensitivity) from the dishcarge.

The magnitude of the laser-induced voltage change across the probes and the absorbed laser power were simultaneously measured for argon. The absorption measurements were made by tuning the wavelength of the laser in a single-mode operation to the atomic transition of interest, and detecting the transmitted laser power by a power-meter (Spectra Physics, 404) with the discharge on and off. The obtained laser-induced voltage change (mV) and the absorbed laser power (mW) for the transition $\left(4 p_{23}-5 d_{34}\right)$ of argon is Tisted in Table I, together with the reported values. From the electron-gas heating mechanism postulated by Keller and Zalewski [12], it is expected that the sensitivity is independent of the atomic transitions except those originating from the metastable states which give very large signais expecially at low discharge current [11]. The obtained sensitivity with the doubleprobe method is one or two orders of magnitude greater than those obtained for nonmetastable states with other methods. The observed high sensitivity can not be explained with the above mechanism alone. The mechanism of the increased efficiency in collecting the OG signals with the double-probe method is not clarified up to now. 
Table I. Extra electrons produced in the external circuit/photon absorbed

\begin{tabular}{|c|c|c|c|c|c|}
\hline & $\lambda(n m)$ & $\Delta V(m V)$ & $I_{a b s}(m W)$ & $\Delta V / I_{a b s}\left(\frac{m V}{m W}\right)$ & $E\left(\frac{\text { electron }}{\text { photon }}\right) \times 10^{4}$ \\
\hline $\mathrm{Ne}^{\mathrm{a}}$ & $618.22\left(2 p_{9}+3 s_{5}\right)$ & & & 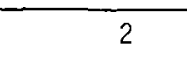 & \\
\hline $\mathrm{Ne}^{\mathrm{a}}$ & $588.19\left(3 \mathrm{P}_{2}+2 \mathrm{p}_{2}\right)$ & & & 46 & \\
\hline $\mathrm{Ne}^{\mathrm{a}}$ & $674.31\left(3 \mathrm{P}_{2} \rightarrow 2 \mathrm{p}_{6}\right)$ & & & 120 & \\
\hline $\mathrm{Ne}^{\mathrm{b}}$ & 614.31 & 506 & 0.11 & 4620 & 220 \\
\hline $\mathrm{Na}^{\mathrm{C}}$ & 589.59 & 0.956 & 0.09 & 11 & 8.4 \\
\hline$U^{c}$ & 591.54 & 33 & 2.7 & 12 & 9.6 \\
\hline$A r^{d}$ & $603.21\left(4 p_{23} \rightarrow 5 d_{34}\right)$ & 42 & 0.07 & 600 & 130 \\
\hline
\end{tabular}

aref. 11

b, cref. 12, ${ }^{c}$ with low discharge current of $0.50 \mathrm{~mA}$

dhis work

Another characteristic of our method is that the $O G$ signals are measurable over a wide pressure range. In He discharge, stable discharge was maintained in the pressure region from 7 Torr to 40 Torr at a microwave power of $20 \mathrm{~W}$. In a higher pressure range beyond 10 Torr, the $O G$ signals of $\mathrm{He}_{2}$ were easily observed around $590 \mathrm{~nm}$ as shown by Fig. 4. The intensity of the $\mathrm{He}_{2}$ transition was found to reach its maximum at a pressure of around 35 Torr.

The observed transitions of $\mathrm{He}_{2}$ are the $f\left({ }^{3} \Sigma_{\mathcal{U}}^{+},{ }^{3} \Pi_{\mathcal{U}},{ }^{3} \Delta_{\mathcal{U}}\right)-b^{3} \Pi_{g}$ bands according to the assignments of emission spectra by Ginter [13]. The triplet splitting have not been resolved for $\mathrm{He}_{2}$ except the partially resolved $b^{3} \mathrm{~m}_{\alpha}$ state [13]. From a Doppler-limited optogalvanic spectrum of the $f^{3} \Sigma_{u}-b^{3} I_{g}$ transition it is found that all the rotational 1 ines except $R(4)$ and $P(4)$ are split into doublets of
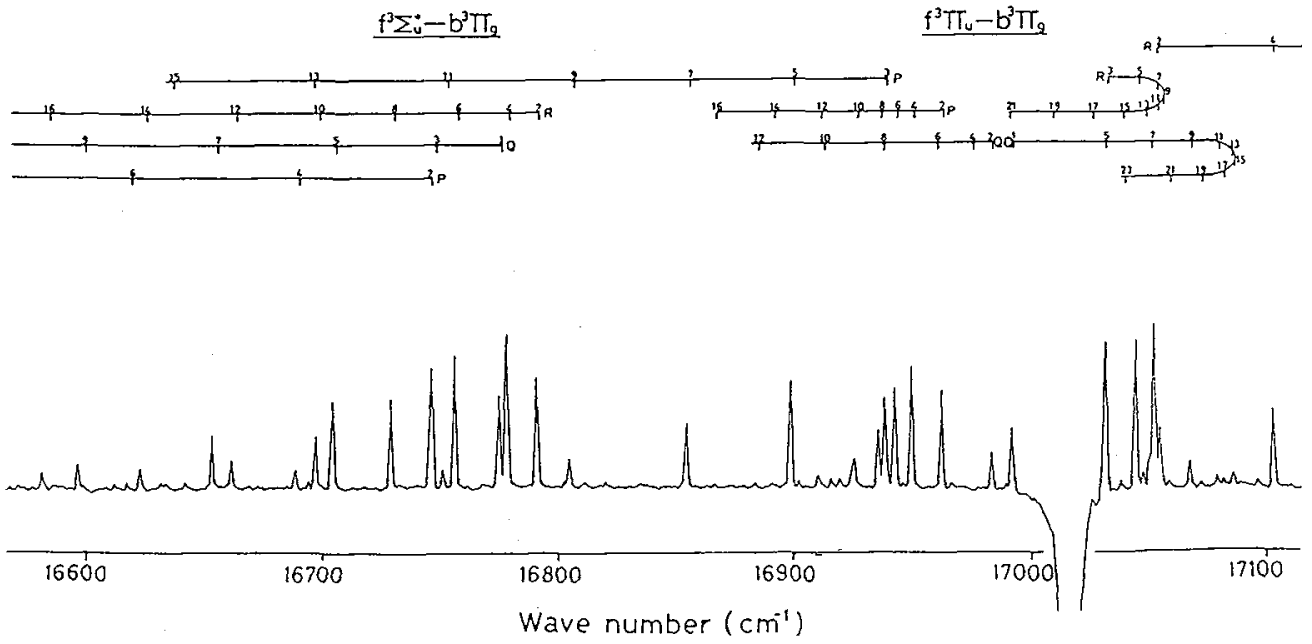

Fig. 4 The optogalvanic spectrum of Hez at a pressure of 35 Torr. Assignments by Ginter[13] are shown according to his emission data.

unequal intensity. The linewidth of the splitted lines is measured to be $3.5 \mathrm{GHz}$ ( FWHM). The corresponding temperature is determined to be $500^{\circ} \mathrm{C}$. Although the homogeneous broadening may have a fairly large contribution to the linewidth of 
$\mathrm{He}_{2}$, the observed transition is broadened mainly by Doppler effect, because the Doppler-width at room temperature is as large as $2.2 \mathrm{GHz}$. This implys a possibi]ity of high resolution study of $\mathrm{He}_{2}$ using a Doppler-free optogalvanic method [5].

3. Mechanism of $O G$ signals with the double probe

A typical view of the discharge region is illustrated in Fig. 5. Because of the inhomogeneous field strength of the microwave inside the resonator, the bright region is not symmetrical between the probes 1 and 2 . In the configuration shown in Fig. 5, the microwave field and the emission from discharge are always more intense at the probe 1 than at the probe 2. At a relatively high pressure, the bright region is restricted to only around the probe 1 at a microwave power of $20 \mathrm{~W}$. This is because the secondary electron emissions at the metal surface supplement the electrons with insufficiently short mean free path to sustain a discharge. As the pressure is decreased, the bright region expands to reach the probe 2 .

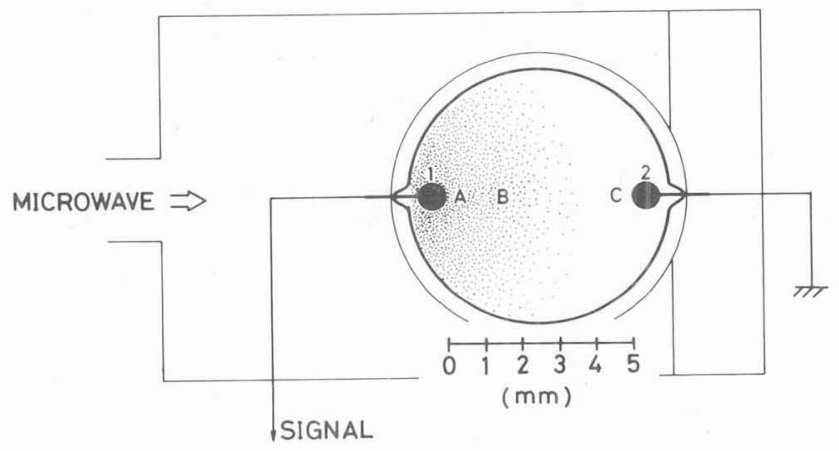

Fig. 5 Schematic drawing of the bright region in the discharge cel1. At a high pressure, the bright region is restricted around the probe 1 . As the pressure decreases, the bright region increases.

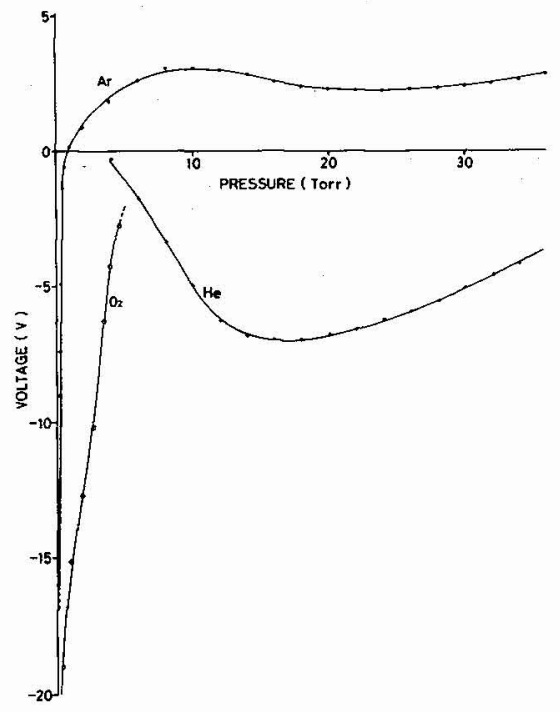

Fig. 6 The pressure dependence of the induced voltage across the two probes in the discharge driven by a 20W microwave. Dotted Tines show unstable discharge region where fine adjustments of cavity condition or microwave power are necessary. 
The pressure dependence of the induced voltage across the probes are shown in Fig. 6 for $\mathrm{Ar}$, He and $\mathrm{O}_{2}$. Here, the induced voltage means the potential at the probe 1 compared to that at the probe 2. The $v-p$ characteristics in argon discharge can be qualitatively understood by the fundamenta] relations in plasma physics [14].

In plasma regions, electrons have a Maxwellian distribution owing to a large collision frequency. Then, the equilibrium relation between pressure and electric field gives the expression of the space potential $V_{s}$ for a constant temperature, as

$$
v_{s}=\frac{k T e}{e} \ln \left(\frac{n_{e}}{n_{e 0}}\right) \text {, }
$$

where $e, k$ and $T e$ are the elementary charge, Boltzmann's constant and the electron temperature, respectively. $n_{e 0}$ is the electron density at an appropriate position where the space potential is taken as zero. For a constant electron density, the space potential is expressed as to be directly proportional to the temperature. Equation (1) says that the electron density increases as the space potential increases. In the discharge condition as shown in Fig. 5, the electron temperature at the probe $1\left(\mathrm{~T}_{e 1}\right)$ is higher than at the probe $2\left(\mathrm{~T}_{e 2}\right)$. Here, the space potential is described not with a constant temperature but with a variable temperature defined in a limited region. Even in this case, eq. (1) can be used as a qualitative description of the overall behavior.

The floating potential $V_{f}$ at the probe surface is decreased by the sheath voltage, and is approximately described by [14]

$$
v_{f}=v_{s}-\alpha \frac{k}{e} T_{e} \text {. }
$$

$\alpha$ is a constant expressed as

$$
\alpha=\frac{1}{2} \ln \left(\frac{\pi}{2} \frac{m}{M}\right),
$$

where $\mathrm{m}, \mathrm{M}$ are the electron and the ion mass respectively. For argon, $\alpha$ is about 6. In argon discharge, the bright region is restricted only around the probe 1 at a high pressure. In this case, the relations of $n_{e l}>>n_{e 2}$ and $T_{e l}>>T_{e} 2$ are expected. When the difference of the space potential between the probe 1 and 2 is 1 arger than the difference of the sheath voltage at the probe 1 and 2 , the observed voltage ( $\left.V_{f 1}-V_{f 2}\right)$ is positive as shown in Fig. 6 . At a low pressure, the bright region spreads across the two probes, where the relations of $n_{e 1} \sim n_{e}$ and $T_{e l}>T_{e} 2$ are expected. In this case, the potential drop at the seath is larger than the difference of the space potential. This explains the observed negative voltage.

The $v-p$ characteristics of other species may also be interpreted by the above considerations when we take into account some specific mechanisms in discharge. In oxygen discharge, relatively large electron affinity of oxygen atom $(1.45 \mathrm{eV})$ or oxygen molecule ( $20.58 \mathrm{ev}$ ) have to be considered. The electron temperature decreases as slow electrons are absorbed to form stable negative ions, but the net charge density is not altered. This effect is significant at the probe 2 where there are less secondary electrons emitted from the metal surface. This process is considered to give the large negative voltage in the oxygen discharge.

In helium discharge, the observed $v-p$ characteristics have a close relation with the signal intensity of the $\mathrm{f}-\mathrm{b}$ transitions of $\mathrm{He}_{2}$. When a $\mathrm{He}_{2}$ molecule in the $b^{3} \Pi_{g}$ state is produced by collisions of an excited He atom with ground state He atoms, an excess energy of at least $1 \mathrm{eV}$ has to be released. This process may result in the increase of the electron temperature around the region where $\mathrm{He}_{2}$ molecules are efficientiy produced. As described before, the OG signals of He2 were detected only around the probe 1. The high electron temperature at the probe 1 will produce a large sheath potential to give a negative voltage shown in the figure. For a more quantitative analysis of the discharge process, plasma parameters $n_{e}$ and $T_{e}$ have to be known. Such analysis is being carried on. 
The spatial dependence of the magnitude and phase of the $O G$ signal was investigated by irradiating the focused laser beam at several points between the two probes, and by simultaneously adjusting the phase of the lock-in amplifier. The results are shown in Fig. 7. The signal amplitude was maximum for the laser excitation close to the surface of the probe 1. A minimum amplitude was obtained by irradiating a region about $1.6 \mathrm{~mm}$ apart from the probe 1. This distance of the minimum amplitude varied with different pressure. Figure 7 shows a rapid phase variation from $0^{\circ}$ to $180^{\circ}$ around this region. With increasing distance from this region, the magnitude of the signal increases gradually while the phase keeps constant. In order to clarify the spatial dependence of the signal, the signals were monitored by an oscilloscope.

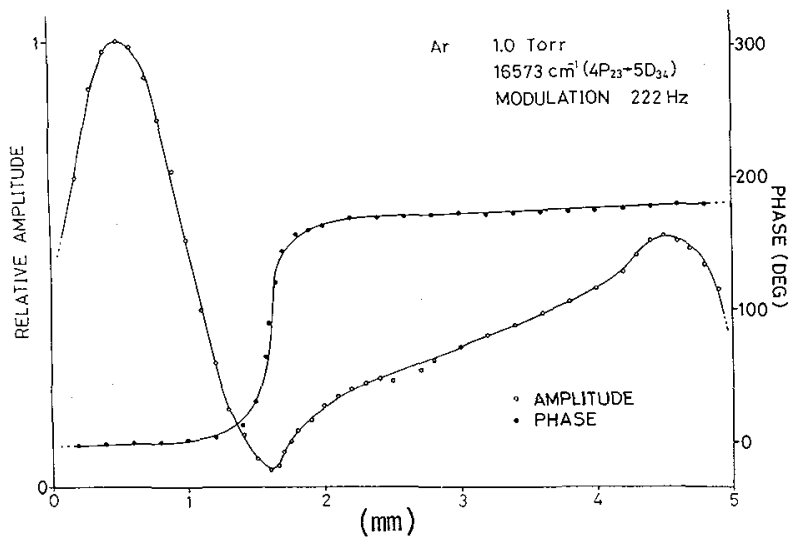

Fig. 7 The variation of the magnitude and phase of the optogalvanic signals with the interelectrode position of laser irradiation. The horizontal scale is the separation between the irradiated position and the probe 1 as shown in Fig. 5 .

Figure 8 shows the oscilloscope traces of the OG signals obtained using a mechanical chopper of $220 \mathrm{~Hz}$. In the figure, (a), (b) and (c) correspond to the laser irradiation at positions A, B and C in Fig. 5. These traces are interpreted as a superposition of a fast (rising and falling) component and a slow component as described below. In trace (a), two positive components are superposed to give a enhanced positive signal, while in trace (b), the sign of the slow component is negative and the superposed signal in the steady state is carcelled out leaving oniy transient responses. In trace (c), the size of the fast component is very small and only the negative slow component is observed.

From these observations shown in Fig. 7 and 8 , mechanisms of the signal generation in the double probes are interpreted as follows: the fast component may be ascribed to the ionization mechanisms either by two-photon transition or by electron impact as has been proposed in many literatures for a hollow cathode discharge. On the other hand, the mechanism of the slow component requires a more complicated description in the plasma process. The energy supplied to the plasma is diffused to the surrounding region through the thermalization process mainiy by the numerous electron collisions, as has been interpreted as the electron gas heating [12]. The thermalization process describes the slow rise and decay in Fig. 8. The increased temperature at the probe 1 caused by laser irradiation at position $A$ increases both the space potential and the sheath potential from eqs. (1) and (2). The net output voltage between the probes in this case is positive as described previously. 


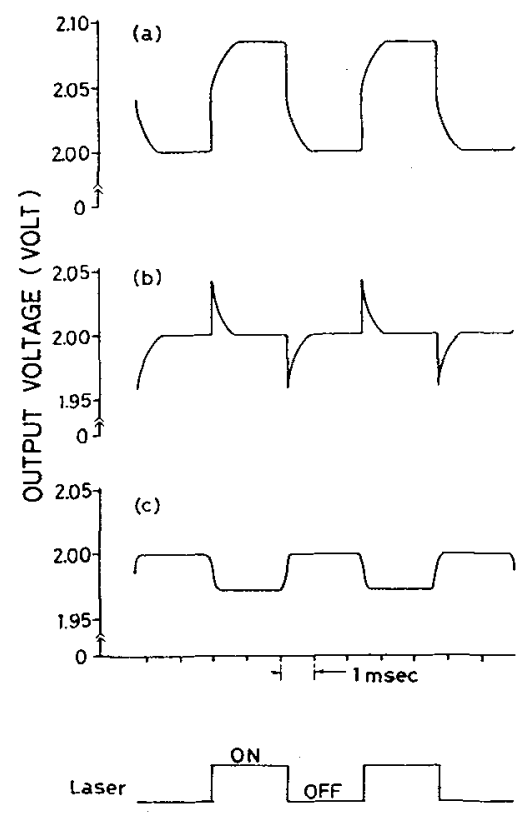

Fig. 8 0scilloscope traces of the optogalvanic signals in Ar, measured with a mechanical chopper of $220 \mathrm{~Hz}$. Traces of (a), (b) and (c) show observed signals generated by laser irradiation at interelectrode positions of A, B and $C$ in Fig. 6 , respectively.

The negative slow component was observed by irradiation over a wide region between $B$ and $C$ in Fig. 5. Negative signals correspond to the increase of the floating potential $V_{f 2}$, because the observed voltage is $\left(V_{f 1}-V_{f 2}\right)$. Although the electron gas-heating mechanism can explain almost the observed behavior, the increase of the net charge density will also contribute to the negative signal because the efficiency of the electron impact ionization process increases with increasing electron temperature [7]. As the charge density at $B$ or $C$ is low, even a small increase in the density will have a large effect on the OG signals at these points. The observed negative signals will be attributed to the combination of the above two mechanisms.

\section{Conctusion}

We have developed a detection technique of the $O G$ signals in the microwave discharge. Although we have to use electrodes (probes) inside the discharge cell in order to obtain a good $\mathrm{S} / \mathrm{N}$ ratio, the double-probe method proved to be very sensitive even with a magnetron which is known to be a noisy oscillator.

Our method is characterized by two distinct features; one is a high efficiency in extracting the OG signals out of discharge and the other is a stable operation at a high pressure. The latter feature will be promissing for the study of excimers, one example of which was shown as a successful observation of $\mathrm{He}_{2}$ molecule. The $O G$ signals in the double-probe method result from at least two main processes, one is the ionization process and the other is electron gas heating. A detailed mechanism of the signal generation in the double probe will be quantitatively interpreted with a further knowledge of the plasma parameters.

\section{Acknowledgements}

The authors are grateful to Professor $K$. Shimoda for the valuable suggestions of the double-probe method and to Dr. Y. Sadamoto for the valuable discussions. 
References

1. Schenck P.K., Mallard W.G., Travis J.C. and Smyth K.C., J. Chem. Phys. 69 (1978) 5147.

2. Feldmann D., Opt. Commun. 29 (1979) 67.

3. Suzuki T., Opt. Commun. 38 (1981) 364.

4. Rettner C.T., Webster C.R. and Zare R.N., J. Phys. Chem. 85 (1981) 1105.

5. Suzuki T. and Kakimoto M., J. Mol. Spectrosc. 93 (1982) $4 \overline{23}$.

6. Vasudev R. and Zare R.N., J. Chem. Phys. 76 (1982) 5267.

7. Webster C.R. and Menzies R.T., J. Chem. Phys. 78 (1983) 2121.

8. Lyons D.R., Schawlow A.L. and Yan G-Y., Opt. Commun. 38 (1981) 35.

9. Brown S.C. in Encyctopedia of physies, Vol. XXII, ed. Flugge S. (SpringerVerlag, 1956) p.537.

10. Labastie P., Biraben F. and Giacobino E., J. Phys. B: At. Mol. Phys. 15 (1982) 2595.

11. Zalewski E.F., Keller R.A. and Engleman R. Jr., J. Chem. Phys. 70 (1979) 1015.

12. Keller R.A. and Zalewski E.F., Appl. Opt. 19 (1980) 3301.

13. Ginter M.L., J. Mo1. SpectrosC. 18 (1965) 321.

14. Chen F.F. in Plasma diagnostic techniques, ed. Huddlestone R.H. and Leonard S.L. TAcademic Press, New York, T965). 